\title{
Sertraline in symptomatic chronic breathlessness: a double blind, randomised trial
}

\author{
David C. Currow ${ }^{1,2}$, Magnus Ekström¹,3, Sandra Louw ${ }^{4}$, Julie Hill ${ }^{4}$, \\ Belinda Fazekas ${ }^{1,2}$, Katherine Clark ${ }^{1,5}$, Patricia M. Davidson ${ }^{1,6}$, \\ Christine McDonald ${ }^{7}$, Dimitar Sajkov ${ }^{8}$, Nikki McCaffrey ${ }^{9}$, Matthew Doogue ${ }^{10}$, \\ Amy P. Abernethy ${ }^{2,11}$ and Meera Agar ${ }^{1}$
}

Affiliations: ${ }^{1}$ IMPACCT, Faculty of Health, University of Technology Sydney, Ultimo, Australia. ${ }^{2}$ Discipline of Palliative and Supportive Services, Flinders University, Adelaide, Australia. ${ }^{3}$ Dept of Clinical Sciences, Division of Respiratory Medicine and Allergology, Lund University, Lund, Sweden. "McCloud Consulting Group, Narabang Way, Belrose, Australia. ${ }^{5}$ University of Sydney, Northern Clinical School, Royal North Shore Hospital, St Leonard's, Australia. 'SChool of Nursing, Johns Hopkins University, Baltimore, MD, USA. ${ }^{7}$ Respiratory and Sleep Medicine, Austin Health, Heidelberg, Australia. ${ }^{8}$ Flinders Medical Centre, Dept of Respiratory and Sleep Medicine, Medical Centre Drive, Bedford Park, Australia. ${ }^{9}$ Deakin Health Economics, School of Health and Social Development, Deakin University, Burwood, Australia. ${ }^{10}$ University of Otago, Christchurch and Canterbury District Health Board, Christchurch, New Zealand. ${ }^{11}$ Department of Medicine, Duke University, Carey, NC, USA.

Correspondence: David C. Currow, IMPACCT, Faculty of Health, University of Technology Sydney, P 0 Box 123, Ultimo, New South Wales, Australia 2007. E-mail david.currowauts.edu.au

@ERSpublications

Chronic breathlessness generates suffering late in life. In this setting, titrated sertraline had similar benefits and harms to placebo in an adequately powered, multi-site, double blind, randomised controlled trial at 4 weeks. http://ow.ly/iWtH30mcMYK

Cite this article as: Currow DC, Ekström M, Louw S, et al. Sertraline in symptomatic chronic breathlessness: a double blind, randomised trial. Eur Respir J 2019; 53: 1801270 [https://doi.org/10.1183/ 13993003.01270-2018].

ABSTRACT Does sertraline provide symptomatic relief for chronic breathlessness in people with advanced disease whose underlying cause(s) are optimally treated?

223 participants with chronic breathlessness (modified Medical Research Council breathlessness scale $\geqslant 2$ ) who had optimal treatment of underlying cause(s) were randomised 1:1 to sertraline 25-100 mg (titrated upwards over 9 days) or placebo for 4 weeks. The primary outcome was the proportion who had an improvement in intensity of current breathlessness $>15 \%$ from baseline on a 100 -mm visual analogue scale.

The proportion of people responding to sertraline was similar to placebo for current breathlessness on days 26-28 (OR 1.00, 95\% CI 0.71-1.40) and for other measures of breathlessness. Quality of life in the sertraline arm had a higher likelihood of improving than in the placebo arm over the 4 weeks (OR 0.21 , 95\% CI 0.01-0.41; $\mathrm{p}=0.044)$. No differences in performance status, anxiety and depression, or survival were observed. Adverse event rates were similar between arms.

Sertraline does not appear to provide any benefit over placebo in the symptomatic relief of chronic breathlessness in this patient population.

This study is registered at www.anzctr.org.au with identifier number ACTRN 12610000464066. Data are available on request from bona fide researchers.

Received: May 242018 | Accepted after revision: Oct 032018

Copyright OERS 2019 


\section{Introduction}

Breathlessness is an uncomfortable sensation of breathing which is experienced by nearly $50 \%$ of people in their last year of life, regardless of diagnosis [1-3]. It is a source of physical and psychological distress for the patient and their caregivers [4].

The main therapy for chronic breathlessness is treating the underlying cause(s). Despite this, many people remain highly symptomatic [5]. Systemic opioids (oral and parenteral) can reduce breathlessness [6, 7]. To date, no other pharmacological intervention has been shown to be of benefit. A meta-analysis of benzodiazepines found no net benefit despite widespread use [8]. Nebulised furosemide and systemic antidepressants are being explored $[9,10]$.

Selective serotonin reuptake inhibitors (SSRIs) are well tolerated antidepressants. Early-phase studies suggest that they may reduce chronic breathlessness, even in people who are not depressed. A potential mechanism is their anxiolytic effect. Sertraline, an SSRI, shows potential benefit for chronic breathlessness, is well tolerated [11] and used first-line as an antidepressant. Two nonrandomised pilot studies and a case series have reported benefits of sertraline $12.5-100 \mathrm{mg}$ in people with chronic breathlessness due to optimally treated chronic obstructive pulmonary disease (COPD) [12-14]. Another study enrolled people with COPD and depression for up to 19 weeks of treatment with paroxetine (another SSRI) in a small double-blind study. People experienced a clinically meaningful improvement in St George's Respiratory Questionnaire scores [15]. In another small randomised trial of 12 weeks of paroxetine in people with COPD, there were significant improvements in the emotional function and mastery domains of the Chronic Respiratory Questionnaire (CRQ) [16]. These data support the need for an adequately powered study.

The aim of this study was to assess the effects of sertraline on intensity of chronic breathlessness despite optimal treatment of underlying cause(s). Secondary aims were to determine the effects of sertraline on quality of life and activities of daily living, and its benefits and harms. The null hypothesis was that there is no difference between sertraline and placebo for chronic breathlessness.

\section{Materials and methods}

Study design

This double-blind, dose increment, parallel-arm, multisite, randomised, placebo-controlled, adaptive study was conducted in accordance with International Conference on Harmonisation Good Clinical Practice [17, 18] and registered at www.anzctr.org.au (ACTRN 12610000464066).

The adaptive design was a blinded phase II/III study where the phase II study was not unblinded (having demonstrated acceptability and feasibility), moving directly to the phase III study. Therefore, phase II data were included in the main analysis.

Participants were recruited from 10 inpatient and outpatient services in palliative care, oncology, respiratory medicine and cardiology units across Australia. Their primary family caregiver was also invited to participate.

\section{Study population}

Potential participants were eligible if they were aged $\geqslant 18$ years; diagnosed with chronic breathlessness where the underlying cause(s) had been maximally treated as attested by their specialist treating physician; had a score $\geqslant 2$ on the modified Medical Research Council (mMRC) breathlessness scale [19]; on stable medications for breathlessness for the previous week (with exception of "as-needed" medications); a life expectancy of $\geqslant 2$ months; and able to read English.

Potential participants were ineligible if they had a previous adverse reaction to sertraline; severe hepatic impairment, gastrointestinal bleeding or respiratory failure; serum sodium $<128 \mathrm{mmol} \cdot \mathrm{L}^{-1}$; uncontrolled seizures; respiratory depression; a respiratory or cardiac event in the past week; severe depression (Hospital Anxiety and Depression Score (HADS) depression subscore $>16$ ); treatment with any medicine that increased the risk of serotonin syndrome; or were pregnant or breastfeeding.

\section{Intervention}

The active study arm was one capsule daily of oral sertraline (days 1-3 $25 \mathrm{mg}$ daily; days 4-6 $50 \mathrm{mg}$ daily; then $100 \mathrm{mg}$ daily). Back-titration was allowed to the next lowest dose if the current dose was not tolerated. Participants could continue on their blinded arm for up to 6 months after completing the first 28 days (primary end-point). At study end, the dose was titrated down, halving the dose every 3 days.

Control

Identical-appearing capsules were administered as a placebo. 
Both arms were permitted to take up to eight doses of $2.5 \mathrm{mg}$ of immediate-release oral morphine solution "as needed" in any 24-h period.

Randomisation (1:1) was stratified by baseline HADS anxiety subscale (0-10: mild to moderate; $11-21$ : moderate to severe) and depression (0-10 and 11-16) giving four strata [20], each within blocks of four per site. Pharmacists allocated participants to the next available code according to a supplied table to dispense identical-appearing sertraline or placebo. Ward pharmacists, investigators, treating clinicians, participants and carers remained blinded to treatment allocation at all times.

At study visits (days 0, 9 and 29), laboratory measures and Australian-modified Karnofsky Performance Status (AKPS) [21] data were collected. Participants completed the CRQ dyspnoea subscale [22], participant and carer quality-of-life questionnaires (European Organisation for Research and Treatment of Cancer (EORTC) core quality-of-life questionnaire (QLQ)-C15-Pal [23] and Caregiver Quality of Life Index - Cancer [24]), HADS [20] and descriptors of breathlessness [25]. Participants completed a diary twice daily (days $0,7,14,21,26,27$ and 28) to record current breathlessness intensity, unpleasantness and worst breathlessness on a $0-100-\mathrm{mm}$ visual analogue scale (VAS) and a four-point Likert scale, and the modified Medical Research Council (mMRC) (table 1) dyspnoea scale [17]. Optionally, participants could remain on blinded treatment for an additional 5 months (6 months treatment in total).

\section{Measurements}

The primary outcome measure was the average of the morning and evening current intensity of breathlessness VAS scores over days 26, 27 and 28. Secondary end-points included exposure to treatment; breathlessness (average, worst and relief over the previous $24 \mathrm{~h}$ and unpleasantness now on $0-100 \mathrm{~mm}$ VAS); response by anxiety and, separately, depression; response in people with and without COPD, response by level of $\mathrm{mMRC}$; use of as-needed immediate-release oral morphine solution; quality of life; CRQ (breathlessness, fatigue, mastery); functional status; anxiety and depression; survival; harms; global impression of change; and choice to enter the extension phase.

\section{Statistical analysis}

Response rates in previous studies of pharmacological interventions for the symptomatic relief of chronic breathlessness have been high [26]. For a new therapy to be considered clinically significant, there must be a substantial net benefit over placebo. By consensus, the expected response rate was set at $41 \%$ for sertraline, while anticipating that $20 \%$ of participants in the placebo arm would improve. A sample size of 75 completed participants per group provided $>80 \%$ power for a two-tailed type 1 error rate of 0.05 between arms. Allowing for expected attrition, recruitment of 240 participants was planned.

Analyses were conducted on an intention-to-treat basis. An interim blinded analysis assessing safety and reviewing the power calculation was conducted by an independent data safety monitoring committee after $50 \%$ of participants completed the study [17].

For the primary analysis, missing values were assumed missing at random and imputed using multiple imputation with 100 samples drawn. Intermittent missing data were imputed using the Markov Chain Monte Carlo procedure. Remaining monotone missing data were imputed using stepwise sequential Bayesian regression.

For the primary end-point, the proportion achieving $>15 \%$ reduction in breathlessness from baseline was compared between groups using the Wald type 3 Chi-squared from a logistic regression model of response with stratum and treatment group as predictor variables. An absolute reduction of $>8.9 \mathrm{~mm}$ VAS over baseline was analysed [27, 28]. Time and group interactions were assessed using longitudinal mixed models with repeated measures (MMRM), with the end-point from days 1-28 as the dependent variable, the baseline value as a covariate, stratum, day, treatment group and treatment group by day interaction as fixed effects and participant as a random effect. A compound symmetry variance-covariance matrix was used. Clinically relevant response predictors were modelled using multivariable regression.

\section{TABLE 1 Modified Medical Research Council dyspnoea scale}

0 Not troubled with breathlessness except on strenuous exercise

1 Short of breath when hurrying on the level or walking up a slight hill

2 Walks slower than contemporaries on level ground because of shortness of breath or has to stop for breath when walking at own pace on the level

3 Stops for breath after walking $\sim 100 \mathrm{~m}$ or after a few minutes on the level

4 Too breathless to leave the house or breathless when dressing or undressing 
Differences in secondary end-points were compared using logistic regression for the response rate end-points (using multiple imputation data), MMRM for the continuous end-points measured over time (using the original data), and analysis of covariance (ANCOVA) for the continuous end-points. Stratum was included as a factor in all statistical models, where strata were a combination of centre, HADS subscale scores and participation in the blinded pilot study. Low-frequency strata were combined using statistical judgement.

All analyses were performed using SAS version 9.4 (SAS Institute, Cary, NC, USA).

The study was approved by relevant human research ethics committees (Cancer Institute NSW 2010C/02/ 120 and Prince of Wales Hospital 13/327HREC/13/POWH/785). All participants provided written, informed consent. Study reporting complies with CONSORT (Consolidated Standards of Reporting Trials) guidelines $[29,30]$.

\section{Results}

Study population

249 people were screened, of whom 223 were randomised (figure 1). Treatment groups were balanced in demographics and baseline clinical characteristics including breathlessness scores (table 2). At baseline 23 (10.3\%) out of 223 had data missing, for which their screening data were used. Data missing for days 26-28 (93 (41.7\%) out of 223) were imputed.

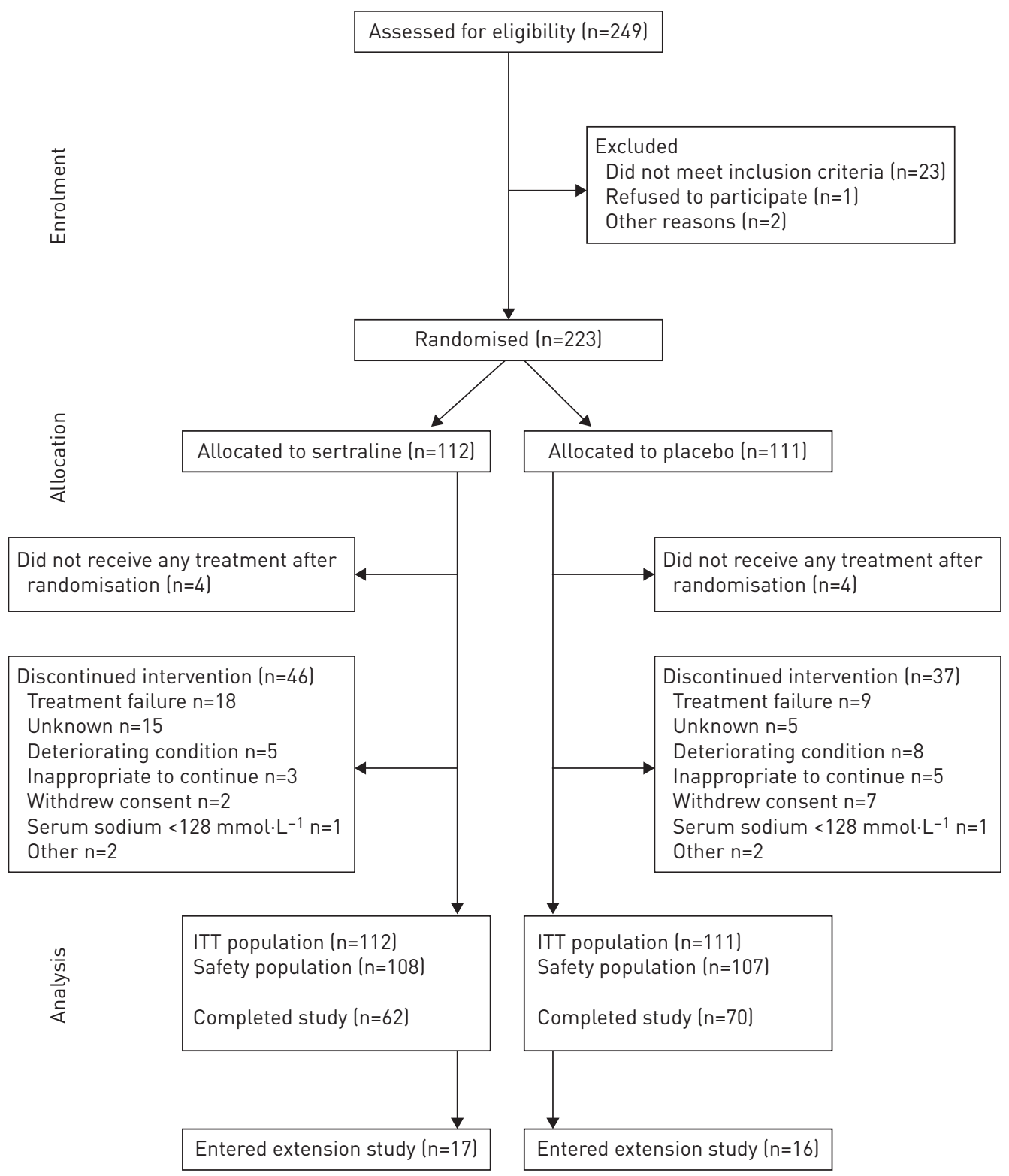

FIGURE 1 Study flow chart. ITT: intention to treat. 
TABLE 2 Demographic and baseline characteristics by treatment group

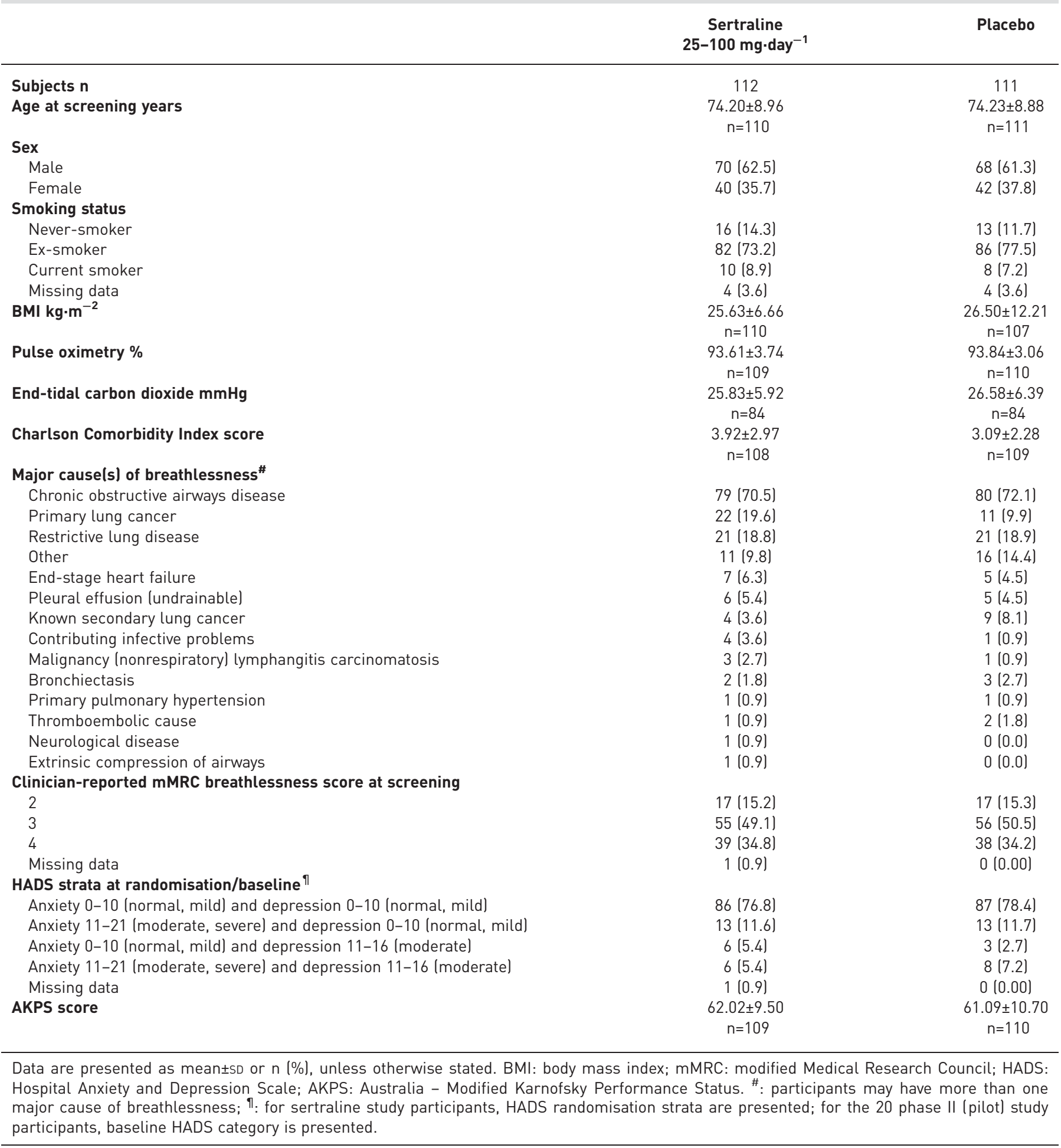

Mean \pm SD exposure to treatment was $38.5 \pm 66.9$ days for sertraline and $55.0 \pm 96.8$ days for placebo. At day 9 (end of titration), 65 participants taking sertraline were on $100 \mathrm{mg}, 10 \mathrm{on} 50 \mathrm{mg}$ and two on $25 \mathrm{mg}$. For placebo, corresponding "doses" were taken by 91, five and two participants, respectively.

Primary outcome

Breathlessness intensity lessened in both arms, with no difference between groups in the primary outcome ( $p=0.636$; figure 2). There was no difference in the proportions with a $15 \%$ reduction in current intensity 

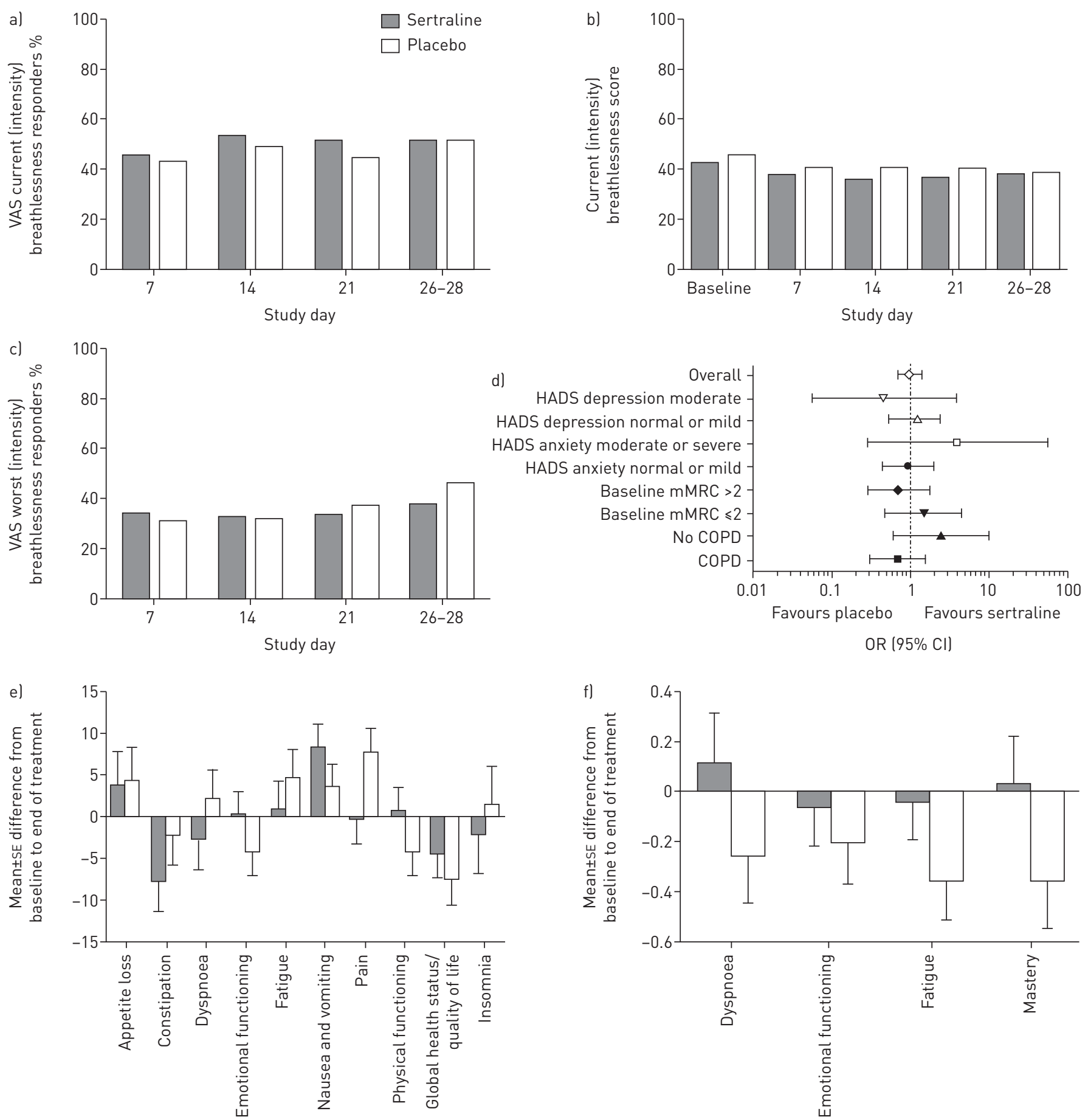

EORTC QLQ15 domains

$\mathrm{CRQ}$ domains

FIGURE 2 Effectiveness data in a double-blind, placebo-controlled trial of sertraline for chronic breathlessness ( $\mathrm{n}=223$ ) for a) proportion of responders with a $15 \%$ reduction in current intensity of breathlessness; b) $0-10 \mathrm{~mm}$ breathlessness intensity scores now; c) 0-100 mm visual analogue scale (VAS) worst breathlessness in the previous $24 \mathrm{~h}$; d) subgroup analysis; e) quality of life; and f) Chronic Respiratory Questionnaire (CRQ). HADS: hospital anxiety and depression scale; mMRC: modified Medical Research Council; COPD: chronic obstructive pulmonary disease; EORTC QLQ: European Organisation for Research and Treatment of Cancer Quality-of-Life Questionnaire.

of breathlessness from baseline (OR 1.00, 95\% CI 0.71-1.40; $\mathrm{p}=0.992$; figure 2). For the same sensation, there was no difference in the proportion achieving $>8.9 \mathrm{~mm}$ reduction (OR 1.02, 95\% CI 0.72-1.44; $\mathrm{p}=0.921)$. In addition, in a model comparing baseline breathlessness to changes over the entire 28 days of the study in people with moderate/severe anxiety accounting rescue medication use, AKPS, Charlson Comorbidity Index (CCI) and pulse oximetry, there was no difference in any breathlessness measure. Sensitivity analyses assessing imputation methods were consistent with these results. 


\section{Secondary outcomes}

There was no difference observed between groups in the proportion of participants who had a $>15 \%$ change from baseline in any measure of breathlessness (figure 2). No subgroup showed any response (those with and without COPD, mMRC score 2 versus 3 or 4 or HADS subscales.

Over the 28-day study period, participants took a similar number of doses of immediate-release oral morphine solution. The mean daily doses taken on days $26-28$ was 0.37 lower in the sertraline arm $(95 \%$ CI $-0.78-0.04, \mathrm{p}=0.080)$.

Mean global quality-of-life scores in the sertraline arm had a greater improvement (days 0-28: 0.21, 95\% CI 0.01-0.41; $\mathrm{p}=0.044$ ) [23] and (days 26-28: 0.64, 95\% CI 0.21-1.07; $\mathrm{p}=0.003$ ). There was a lower pain domain score for sertraline in the EORTC-QLQ-15 [23] $(-8,95 \%$ CI $-14--2 ; \mathrm{p}=0.015$; figure 2). For the $\mathrm{CRQ}$, there was a consistent direction of benefit in the sertraline group for three domains favouring sertraline: breathlessness $(0.36,95 \% \mathrm{CI}-0.07-0.80 ; \mathrm{p}=0.098)$; fatigue $(0.32,95 \% \mathrm{CI}-0.03-0.66 ; \mathrm{p}=0.070)$; and mastery $(0.38,95 \%$ CI $0.00-0.77 ; \mathrm{p}=0.051)$ [22].

At the end of the study, 26 (36.1\%) out of 72 participants on sertraline and $31(41.3 \%)$ out of 75 on placebo felt appreciable improvement $(\mathrm{p}=0.6587)$. A minority felt sufficient benefit for long-term use (sertraline $18.6 \%$, placebo $26.3 \%$ ) and fewer entered the blinded extension study (sertraline 17.3\%, placebo 16.3\%; nonsignificant). (Pilot study participants did not have the option of the extension study) [18].

There were no difference in functional status, nor anxiety and depression scores. Using baseline values for carbon dioxide, mMRC, CCI, HADS anxiety, rescue medication use and AKPS as predictors of breathlessness response $(\geqslant 8.9 \mathrm{~mm}$ change), there was only one significant association: a higher Charlson Comorbidity Index was associated with a greater likelihood of response for VAS average breathlessness in the previous $24 \mathrm{~h}$ alone $(\mathrm{p}=0.041)$ and with the other prognostic factors $(0.028)$.

There were 30 deaths reported in the sertraline group, and 20 reported in the placebo group. Median survival was similar (sertraline 184 days, 95\% CI 96-216 days, placebo 167 days, 95\% CI 117-not reached).

\section{Adverse events}

The majority of participants in each treatment group experienced at least one treatment-emergent adverse event (TEAE) (sertraline 95.4\%, placebo 90.7\%; table 3). 24 (22.2\%) participants in the sertraline group and $34(31.8 \%)$ in the placebo group experienced serious TEAEs. In the sertraline and placebo arms, the serious events experienced by the most participants were exacerbations of COPD (5.6\% and $8.4 \%$, respectively) and breathlessness (2.8\% and 5.6\%, respectively). No subjects experienced hyponatraemia.

\section{Discussion}

There was no net benefit observed for sertraline on chronic breathlessness in participants who had undergone optimal treatment of the underlying cause(s) in this multisite randomised controlled trial. This contrasts with very limited previous evidence that suggested potential benefit [12-14]. In this study, randomisation was stratified by underlying severity of depression and anxiety, and the majority of participants (nearly $80 \%$ in each group) had no or minimal features of anxiety or depression (table 2).

These results are consistent with one study that found no effect of SSRIs in the management of cancer-related breathlessness [14]. The outcomes of the phase 2 BETTER-B study which is evaluating mirtazapine for this indication are awaited with interest (EudraCT 2015-006064-11).

Potential benefits of sertraline were seen for global quality of life, pain, fewer doses of as-needed immediate-release morphine solution and CRQ subscales. Any differences, if real, are small and not clinically significant. These signals should be interpreted with caution given multiple testing of a large number of secondary end-points. Measures of blinded preference or continuing onto the extension study reflected no perceived net benefit of sertraline by participants.

Mastery was a positive outcome in the landmark study by HigGINSON et al. [31] of an integrated palliative and respiratory care support service for chronic breathlessness. This questions the ideal end-point in symptom studies when wellbeing and a sense of control are positive outcomes, but symptom scores do not change. For example, people may exert themselves to the same level of breathlessness (and thus report no better symptom control), but be able to function more effectively.

Sertraline is registered for the treatment of social anxiety disorder [12]. The largest double-blind, pharmacological randomised controlled trial for chronic breathlessness evaluated buspirone, an anxiolytic [32]. That study of people with chronic breathlessness receiving chemotherapy was strongly negative. The role of anxiety in chronic breathlessness is not well defined, noting that a consecutive case series of 100 people 
TABLE 3 Grade 3, 4 or 5 treatment-emergent adverse events of special interest by treatment group

\begin{tabular}{|c|c|c|}
\hline & $\begin{array}{c}\text { Sertraline } \\
\text { 25-100 mg·day }{ }^{-1}\end{array}$ & Placebo \\
\hline Subjects $\mathrm{n}$ & 108 & 107 \\
\hline Participants with at least one adverse event & $12(11.1)$ & $13(12.1)$ \\
\hline Gastrointestinal disorders & $2(1.9)$ & $3(2.8)$ \\
\hline Dry mouth & $1(0.9)$ & $0(0.0)$ \\
\hline Nausea & $1(0.9)$ & $3(2.8)$ \\
\hline Injury, poisoning and procedural complications & $2(1.9)$ & $3(2.8)$ \\
\hline Falls & $2(1.9)$ & $3(2.8)$ \\
\hline Nervous system disorders & $3(2.8)$ & $5(4.7)$ \\
\hline Dizziness & $0(0.0)$ & 4 (3.7) \\
\hline Somnolence & $2(1.9)$ & $2(1.9)$ \\
\hline Tremor & $1(0.9)$ & $2(1.9)$ \\
\hline Psychiatric disorders & $4(3.7)$ & $4(3.7)$ \\
\hline Agitation & $1(0.9)$ & $2(1.9)$ \\
\hline Insomnia & $3(2.8)$ & $1(0.9)$ \\
\hline Restlessness & $2(1.9)$ & $3(2.8)$ \\
\hline Reproductive system and breast disorders & $1(0.9)$ & $0(0.0)$ \\
\hline Ejaculation disorder & $1(0.9)$ & $0(0.0)$ \\
\hline Vascular disorders & $0(0.0)$ & $1(0.9)$ \\
\hline Hypertension & $0(0.0)$ & $1(0.9)$ \\
\hline \multicolumn{3}{|c|}{$\begin{array}{l}\text { Data are presented as } \mathrm{n}(\%) \text {, unless otherwise stated. Investigator text for adverse events encoded using } \\
\text { MedDRA (version } 19.1 ; \text { www.meddra.org). Treatment-emergent adverse events are adverse events that } \\
\text { started or worsened after baseline. }\end{array}$} \\
\hline
\end{tabular}

with cancer and breathlessness found that one person had anxiety as the only cause [33]. Future research needs to differentiate anxiety levels between acute and chronic breathlessness.

The majority of participants in the current study did not have high levels of anxiety or depression HADS scores. Given the relatively small proportion of people with higher levels of anxiety, there may be a case for further studies in this subgroup.

To date, the best quality evidence for relief of chronic breathlessness remains with morphine [6, 7], and concerns around the potentially rare but catastrophic side-effect of respiratory depression are slowly abating, primarily due to evidence that regular, low-dose, extended-release morphine, which minimises peak drug concentrations, does not appear to be associated with this effect in steady state $[6,7,34,35]$.

Limitations in the study include that it was a requirement of the human research ethics committee that immediate-release oral morphine solution be made available to all participants, potentially masking benefits from, and attributing harms to the study medication. There was no end-point assessing changes in function, such as accelerometry. The study duration was relatively long for frail people, and so completion rates are lower than in well participants.

The current findings do not support the use of sertraline for the symptomatic treatment of chronic breathlessness, nor does this study identify any subgroup for further study where there is signal of potential benefit using baseline clinicodemographic data.

Conflict of interest: D.C. Currow has received an unrestricted research grant from Mundipharma, is an unpaid member of an advisory board for Helsinn Pharmaceuticals and has consulted to Mayne Pharma and received intellectual property payments from them. He is a paid consultant to Specialist Therapeutics. M. Ekström has nothing to disclose. S. Louw reports personal fees for statistic consultation from Palliative Care Clinical Studies Collaborative, during the conduct of the study. J. Hill has nothing to disclose. B. Fazekas has nothing to disclose. K. Clark has nothing to disclose. P.M. Davidson has nothing to disclose. C. McDonald reports personal fees for speaking from GSK and Novartis, personal fees for advisory board work from Pfizer, and fees for speaking that were paid directly to the hospital from Menarini, outside the submitted work. D. Sajkov has nothing to disclose. N. McCaffrey has nothing to disclose. M. Doogue has nothing to disclose. A.P. Abernethy has nothing to disclose. M. Agar has nothing to disclose.

Support statement: This study was supported by the Australian Government Commonwealth Department of Health thought the National Palliative Care Program and National Health and Medical Research Council project grant GNT1010065, and sponsored by Flinders University. Funding information for this article has been deposited with the Crossref Funder Registry. 


\section{References}

1 Reuben DB, Mor V. Dyspnea in terminally ill cancer patients. Chest 1986; 89: 234-236.

Ripamonti C. Management of dyspnea in advanced cancer patients. Support Care Cancer 1999; 7: 233-243.

Bruera E, Schmitz B, Pither J, et al. The frequency and correlates of dyspnea in patients with advanced cancer. J Pain Symptom Manage 2000; 19: 357-362.

4 O'Driscoll M, Corner J, Bailey C. The experience of breathlessness in lung cancer. Eur J Cancer Care 1999; 8: 37-43.

5 Johnson MJ, Yorke J, Hansen-Flaschen J, et al. Towards an expert consensus to delineate a clinical syndrome of chronic breathlessness. Eur Respir J 2017; 49: 160277.

6 Ekström M, Bajwah S, Bland JM, et al. One evidence base; three stories: do opioids relieve chronic breathlessness? Thorax 2018; 73: 88-90.

7 Ekström M, Nilsson F, Abernethy AA, et al. Effects of opioids on breathlessness and exercise capacity in chronic obstructive pulmonary disease. A systematic review. Ann Am Thorac Soc 2015; 12: 1079-1092.

8 Simon ST, Higginson IJ, Booth S, et al. Benzodiazepines for the relief of breathlessness in advanced malignant and non-malignant diseases in adults. Cochrane Database Syst Rev 2016; 10: CD007354.

9 Newton PJ, Davidson PM, Macdonald P, et al. Nebulized furosemide for the management of dyspnea: does the evidence support its use? J Pain Symptom Manage 2008; 36: 424-441.

10 Better Treatments for Refractory Breathlessness. https://doi.org/10.1186/ISRCTN32236160. Date last accessed: April 29, 2018. Date last updated January 30, 2017.

11 Rayner L, Price A, Evans A, et al. Antidepressants for the treatment of depression in palliative care: systematic review and meta-analysis. Palliat Med 2011; 25: 36-51.

12 Papp LA, Weiss JR, Greenberg HE, et al. Sertraline for chronic obstructive pulmonary disease and comorbid anxiety and mood disorders. Am J Psychiatry 1995; 152: 1531.

13 Smoller JW, Pollack MH, Systrom D, et al. Sertraline effects on dyspnea in patients with obstructive airways disease. Psychosomatics 1998; 39: 24-29.

14 Perna G, Cogo R, Bellodi L. Selective serotonin re-uptake inhibitors beyond psychiatry: are they useful in the treatment of severe, chronic, obstructive pulmonary disease? Depress Anxiety 2004; 20: 203-204.

15 Eiser N, Harte R, Spiros K, et al. Effect of treating depression on quality-of-life and exercise tolerance in severe COPD. COPD 2005; 2: 233-241.

16 Lacasse Y, Beaudoin L, Rousseau L, et al. Randomized trial of paroxetine in end-stage COPD. Monaldi Arch Chest Dis 2004; 61: 140-147.

17 International Conference on Harmonisation of Technical Requirements for Registration of Pharmaceuticals for Human Use. Guideline for Good Clinical Practice ICH Harmonised Tripartite 1996. Available from: www.ich.org/ fileadmin/Public_Web_Site/ICH_Products/Guidelines/Efficacy/E6/E6_R1_Guideline.pdf

18 Watts GJ, Clark K, Agar M, et al. Study protocol: a phase III randomised, double-blind, parallel arm, stratified, block randomised, placebo-controlled trial investigating the clinical effect and cost-effectiveness of sertraline for the palliative relief of breathlessness in people with chronic breathlessness. BMJ Open 2016; 6: e013177.

19 Bestall JC, Paul EA, Garrod R, et al. Usefulness of the Medical Research Council (MRC) dyspnoea scale as a measure of disability in patients with chronic obstructive pulmonary disease. Thorax 1999; 54: 581-586.

20 Zigmond AS, Snaith RP. The hospital anxiety and depression scale. Acta Psychiatr Scand 1983; 67: 361-370.

21 Abernethy AP, Shelby-James T, Fazekas B, et al. The Australian-modified Karnofsky Performance Status (AKPS) scale: a revised scale for contemporary palliative care clinical practice. BMC Palliat Care 2005; 4: 7.

22 American Thoracic Society. Chronic Respiratory Disease Questionnaire - CRQ. http://qol.thoracic.org/sections/ instruments/ae/pages/crq.html Date last accessed: December 15, 2017. Date last updated: April 2002.

23 Kaasa S, Bjordal K, Aaronson N, et al. The EORTC core quality of life questionnaire (QLQ-C30): validity and reliability when analysed with patients treated with palliative radiotherapy. Eur J Cancer 1995; 31A: 2260-2263.

24 Weitzner MA, Jacobsen PB, Wagner HJ, et al. The Caregiver Quality of Life Index-Cancer (CQOLC) scale: development and validation of an instrument to measure quality of life of the family caregiver of patients with cancer. Qual Life Res 1999; 8: 55-63.

25 Wilcock A, Crosby V, Hughes A, et al. Descriptors of breathlessness in patients with cancer and other cardiorespiratory diseases. J Pain Symptom Manage 2002; 23: 182-189.

26 Currow DC, Ekström M, Fazekas B, et al. A phase III, multi-site, randomised, double blind, placebo controlled parallel arm study of daily extended release (ER) morphine for chronic breathlessness. Eur Respir J 2016; 48: OA4808.

27 Johnson MJ, Bland JM, Oxberry S, et al. Measuring improvement in dyspnoea: should absolute or relative values be used? Eur Resp J 2014; 44: 1700-1703.

28 Johnson MJ, Bland JM, Oxberry SG, et al. Clinically important differences in the intensity of chronic refractory breathlessness. J Pain Symptom Manage 2013; 46: 957-963.

29 Schulz KF, Altman DG, Moher D. CONSORT 2010 statement: updated guidelines for reporting parallel group randomised trials. BMJ 2010; 340: c332.

30 Moher D, Hopewell S, Schulz KF, et al. CONSORT 2010 explanation and elaboration: updated guidelines for reporting parallel group randomised trials. BMJ 2010; 340: c869.

31 Higginson IJ, Bausewein C, Reilly CC, et al. An integrated palliative and respiratory care service for patients with advanced disease and refractory breathlessness: a randomised controlled trial. Lancet Respir Med 2014; 2: 979-987.

32 Peoples AR, Bushunow PW, Garland SN, et al. Buspirone for management of dyspnea in cancer patients receiving chemotherapy: a randomized placebo-controlled URCC CCOP study. Support Care Cancer 2016; 24: 1339-1347.

33 Dudgeon DJ, Lertzman M. Dyspnea in the advanced cancer patient. J Pain Symptom Manage 1998; 16: $212-219$.

34 Abernethy AP, Currow DC, Frith P, et al. Randomised, double blind, placebo controlled crossover trial of sustained release morphine for the management of refractory dyspnoea. BMJ 2003; 327: 523-528.

35 Verberkt CA, van den Beuken-van Everdingen MHJ, Schols JMGA, et al. Respiratory adverse effects of opioids for breathlessness: a systematic review and meta-analysis. Eur Respir J 2017; 50: 1701153. 